\title{
Motion Coordination using Virtual Nodes
}

\author{
Nancy Lynch, Sayan Mitra, and Tina Nolte \\ CSAIL, MIT \\ 32 Vassar Street \\ Cambridge, MA 02139, USA \\ \{lynd,mitras, tnolte $\quad$ Cecsail.mit.edu
}

\begin{abstract}
We describe how a virtual node abstraction layer can be used to coordinate the motion of real mobile nodes in a region of 2 -space. In particular, we consider how nodes in a mobile ad hoc network can arrange themselves along a predetermined curve in the plane, and can maintain themselves in such a configuration in the presence of changes in the underlying mobile ad hoc network, specifically, when nodes may join or leave the system or may fail. Our strategy is to allow the mobile nodes to implement a virtual layer consisting of mobile client nodes, stationary Virtual Nodes (VNs) at predetermined locations in the plane, and local broadcast communication. The VNs coordinate among themselves to distribute the client nodes relatively evenly among the VNs' regions, and each $\mathrm{VN}$ directs its local client nodes to form themselves into the local portion of the target curve.

Index Terms-Motion coordination, virtual nodes, hybrid systems, hybrid I/O automata.
\end{abstract}

\section{INTRODUCTION}

Motion coordination is the general problem of achieving some global spatial pattern of movement in a set of autonomous agents. An important motivation for studying distributed motion coordination, that is, coordination among agents with only local communication ability and therefore limited knowledge about the state of the entire system, stems from the developments in the field of mobile sensor networks. Previous work in this area includes different coordination goals, for example: flocking [9], rendezvous [1], [10], [13], deployment [2], pattern formation [15], and aggregation [7]. Owing to the intrinsic decentralized nature of sensor network applications like surveillance, search and rescue, monitoring, and exploration, centralized or leader based approaches are ruled out. However, the lack of central control makes the programming task quite difficult.

In prior work [3], [4], [5], [6], we have developed a notion of "virtual nodes" for mobile ad hoc networks. A virtual node is an abstract, relatively well-behaved active node that is implemented using less well-behaved real nodes. Virtual nodes can be used to solve problems such as providing atomic memory [4], geographic routing [3], and point-topoint routing [6].

In this paper, we explore the use of virtual nodes in solving motion coordination problems. Namely, we con-

*Research supported by AFRL contract number F33615-010C-1850, DARPA/AFOSR MURI contract number F49620-02-1-0325, NSF ITR contract number CCR-0121277, and DARPA-NEST contract number F33615-01-C-1896. sider virtual nodes associated with predetermined, welldistributed locations in the plane, communicating among themselves and with mobile "client nodes" using local broadcast. We describe one way of implementing such virtual nodes using the real mobile nodes, and describe how such virtual nodes can be used to solve a simple motion coordination problem. We use the Hybrid I/O Automata (HIOA) mathematical framework [11] for describing the components in our systems.

The paper is organized as follows: Section II describes the underlying mobile network. Section III describes our virtual node layer. Section IV defines the motion coordination problem we consider. Section V describes an algorithm for solving this motion coordination problem using the virtual node layer. Section VII gives the proofs of correctness of the algorithm. Section VII outlines one way to implement the virtual node layer, and Section VIII concludes.

\section{THE PHYSICAL LAYER}

Our physical model of the system consists of a finite but unknown number of communicating physical nodes in a bounded square $\mathcal{B}$ in $R^{2}$. We assume that each node has a unique identifier from a set $\mathcal{I}$. Formally, our physical layer model consists of three types of HIOA (see Figure 1): (1) automata $P N_{i}$ to model physical nodes with identifiers $i \in$ $\mathcal{I}$, (2) a $L B$ cast automaton that models the local broadcast communication service between the physical nodes, and (3) a "real world" automaton $R W$ to model the physical location of all the nodes and the real time.

Figure 2 shows the required components of each automaton $P N_{i}$; it may have other internal variables (initially set to unique initial values) and actions, which are not specified here. $P N_{i}$ continuously receives from $R W$ the current time as the input variable realtime and its position as the input variable $\mathbf{x}_{i}$, and communicates its velocity to $R W$ through the output variable $\mathbf{v}_{i}$. The speed of $P N_{i}$ is bounded by $v_{c}$. The trajectories of the continuous variable $\mathbf{v}_{i}$ and the effects of the send and receive actions are unspecified. At each point $P N_{i}$ is either in active or inactive mode; we assume that, initially, finitely many nodes are active. The fail ${ }_{i}$ input action sets the mode to inactive and the recover $_{i}$ input action sets it to active. In inactive mode, all internal and output actions are disabled, no input action except recover ${ }_{i}$ affects the internal or output variables, and during trajectories, the locally-controlled variables remain 


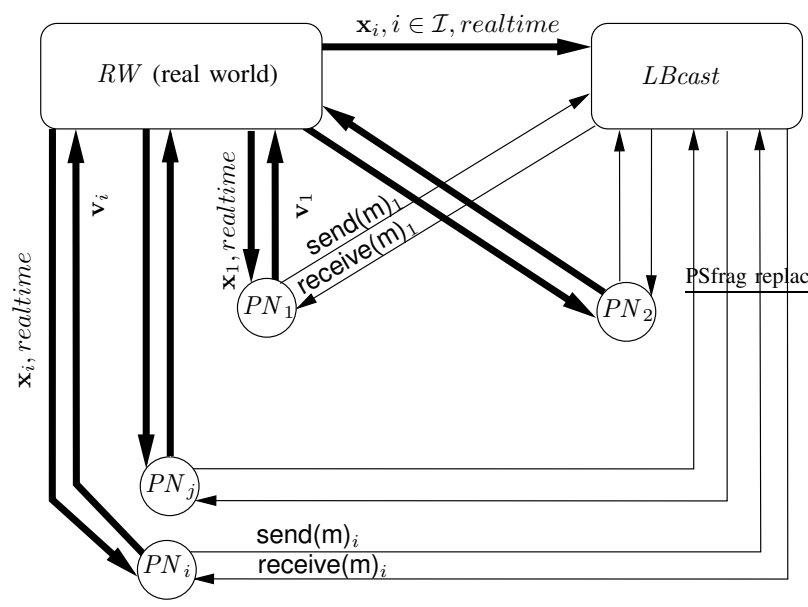

Fig. 1. The Physical Layer: $P N$ automata communicate with each other through an LBcast service and receive time and location information continuously from $R W$.

constant and the velocity $\mathbf{v}_{i}$ remains zero. Thus, we assume that, in inactive mode, $P N_{i}$ stops moving. We model the departure of a node from $\mathcal{B}$ as a failure. For convenience, we assume that transitions are instantaneous.

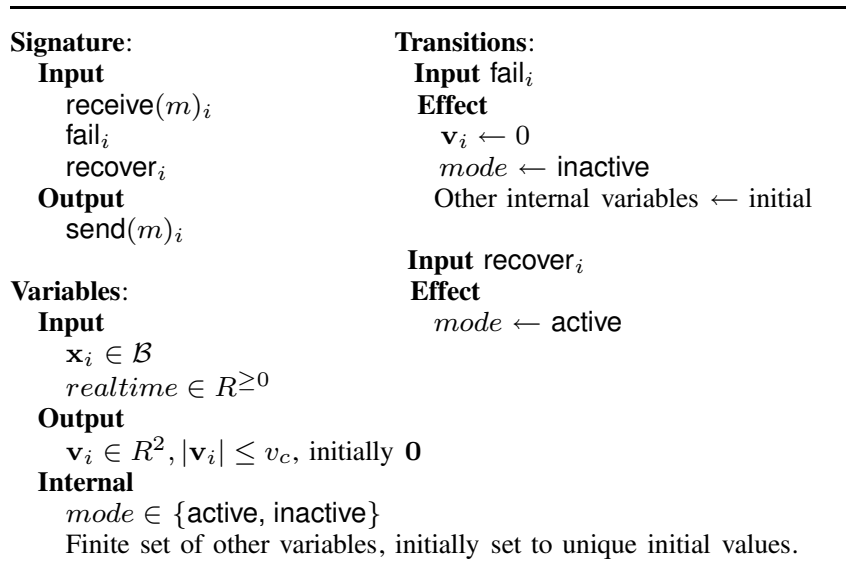

Fig. 2. Hybrid I/O Automaton $P N_{i}$.

The $P N$ s communicate using a local broadcast service, LBcast, which is a generic local broadcast service parameterized by a radius $R_{p}$ and a maximum message delay $d_{p}$. The $L B \operatorname{cast}\left(R_{p}, d_{p}\right)$ service guarantees that when $P N_{i}$ performs a send $(m)_{i}$ action at some time $t$, the message is delivered within the interval $\left[t, t+d_{p}\right]$, by a receive $(m)_{j}$ action, to every $P N_{j}$ that remains in active mode and within $R_{p}$ distance of $P N_{i}$ for the entire interval $\left[t, t+d_{p}\right]$.

The $R W$ automaton (see Figure 3 ) reads the velocity output $\mathbf{v}_{i}$ from each $P N_{i}, i \in \mathcal{I}$, and produces the position $\mathbf{x}_{i}$ for $P N_{i}$ and the LBcast automaton. LBcast requires the node position information because it guarantees delivery only between "nearby" nodes. $R W$ also produces realtime for all physical layer components.

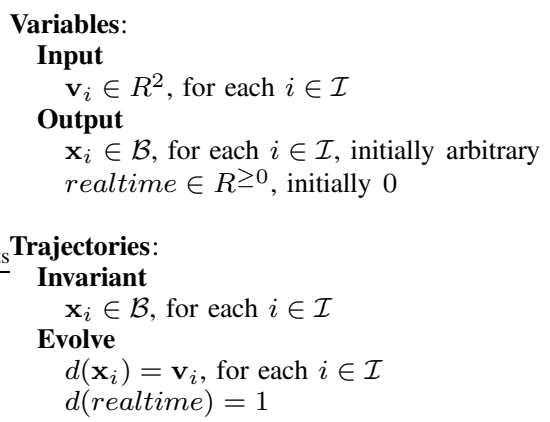

Fig. 3. $R W$ automaton.

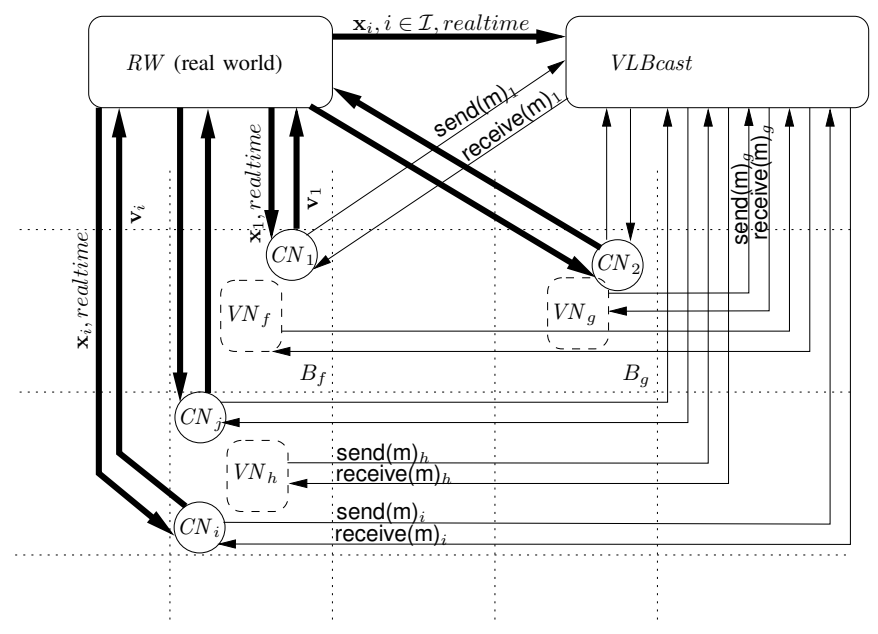

Fig. 4. Virtual Node Layer: $V N \mathrm{~s}$ and $C N \mathrm{~s}$ communicate using the $V L B$ cast service.

\section{The VirTual LAYeR}

The bounded square $\mathcal{B}$ is partitioned into a finite set of zones $B_{h}, h \in \mathcal{H}$. For simplicity we assume $\mathcal{B}$ is a $m \times m$ square grid, with each grid square corresponding to a zone and having sides of length $b$. Each boundary point of a square is unambiguously assigned to one zone. The index set $\mathcal{H}$ is the set of coordinates of the centers of all squares. For each $B_{h}$, the set $\mathrm{Nbrs}_{h}$ contains the zone identifiers of the north, south, east, and west neighboring grid squares.

Our virtual layer abstraction (see Figure 4) consists of: (1) client node automata $C N_{i}$ with identifiers $i \in \mathcal{I}$, (2) one stationary virtual node automaton $V N_{h}$ for each $h \in \mathcal{H}$, located at the center $\mathbf{o}_{h}$ of the square $B_{h}$, (3) a virtual communication service, VLBcast $=\operatorname{LBcast}\left(R_{v}, d_{v}\right)$, for the $V N \mathrm{~s}$ and the $C N \mathrm{~s}$, and (4) an automaton $R W$ to model the physical location of all the $C N \mathrm{~s}$ and the real time.

A client node automaton $C N_{i}, i \in \mathcal{I}$, is a portion of a $P N_{i}$ automaton that has the input variables realtime and $\mathbf{x}_{i}$ from the $R W$ automaton and an output variable $\mathbf{v}_{i}$ to the $R W$ automaton. With respect to failures, an automaton $C N_{i}$ behaves the same as $P N_{i} . C N_{i}$ also has send and receive actions for interacting with the VLBcast service. 
A virtual node automaton $V N_{h}, h \in \mathcal{H}$, is an MMT automaton [12], [14] parameterized by a time upper bound, $d_{M M T}$; it has no realtime clock variable. MMT automata are discrete I/O automata that have a "task" structure, which is an equivalence relation on the set of locally-controlled actions, such that from a point in an execution where a task becomes enabled, within at most time $d_{M M T}$, some action in that task must occur. $V N_{h}$ can fail, disabling internal and output actions, preventing any inputs other than recover $_{h}$ from resulting in state changes, and setting the automaton

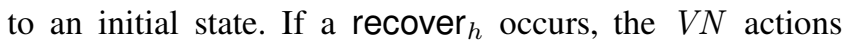
become enabled with all tasks restarted. If $V N_{h}$ is failed and a $C N$ later enters $B_{h}$ and remains active in the zone for $d_{r}$ time, then a recover ${ }_{h}$ occurs within that $d_{r}$ time. $V N_{h}$ communicates with other $V N \mathrm{~s}$ and $C N \mathrm{~s}$ using the $V L B$ cast service through send $_{h}$ and receive ${ }_{h}$ actions.

$V L B$ cast is an LBcast service (as described in the physical layer) for the virtual layer, parameterized by radius $R_{v}$ and maximum message delay $d_{v}$, where $R_{v} \geq b$. It allows $V N_{h}$ to communicate with the $V N \mathrm{~s}$ in the set $N_{b r} s_{h}$ and with $C N s$ that are located in $B_{h}$. It does not allow $C N$ automata to communicate with one another.

The $R W$ automaton in the virtual layer is similar to the one in the physical layer, but here it communicates (through the realtime and $\mathrm{x}$ variables) only with the $C N$ automata and the VLBcast automaton, and not the $V N$ automata.

This virtual layer will be used in Section V to implement a solution to the distributed motion coordination problem. Details of how this virtual layer can be implemented using the physical layer are in Section VII. There we further discuss the relation between the parameters $d_{M M T}, d_{r}, d_{v}$, and $R_{p}$, the physical layer broadcast radius.

\section{The Motion Coordination Problem}

A differentiable parameterized curve $\Gamma$ is a differentiable map $P \rightarrow \mathcal{B}$, where the domain set $P$ of parameter values is an interval in the real line. The curve $\Gamma$ is regular if for every $p \in P,\left|\Gamma^{\prime}(p)\right| \neq 0$. For $a, b \in P$, the arc length of a regular curve $\Gamma$ from $a$ to $b$, is given by $s(\Gamma, a, b)=$ $\int_{a}^{b}\left|\Gamma^{\prime}(p)\right| d p . \Gamma$ is said to be parameterized by arc length if for every $p \in P,\left|\Gamma^{\prime}(p)\right|=1$. For a curve parameterized by arc length, $s(\Gamma, a, b)=b-a$.

For a given point $\mathbf{x} \in \mathcal{B}$, if there exists $p \in P$ such that $\Gamma(p)=\mathbf{x}$, then we say that the point $\mathbf{x}$ is on the curve $\Gamma$; abusing the notation, we write this as $x \in \Gamma$. We say that $\Gamma$ is a simple curve provided for every $\mathbf{x} \in \Gamma, \Gamma^{-1}(\mathbf{x})$ is unique. A sequence $\mathbf{x}_{1}, \ldots, \mathbf{x}_{n}$ of points in $\mathcal{B}$ are said to be evenly spaced on a curve $\Gamma$ if there exists a sequence of parameter values $p_{1}<p_{2} \ldots<p_{n}$, such that for each $i, 1 \leq i \leq n, \Gamma\left(p_{i}\right)=\mathbf{x}_{i}$, and for each $i, 1<i<n$, $p_{i}-p_{i-1}=p_{i+1}-p_{i}$.

In this paper we fix $\Gamma$ to be a simple, differentiable curve that is parameterized by arc length. Let $P_{h}=\{p \in P$ : $\left.\Gamma(p) \in B_{h}\right\}$ be the domain of $\Gamma$ in zone $B_{h} \subset \mathcal{B}$. The local part of the curve $\Gamma$ in zone $B_{h}$ is the restriction $\Gamma_{h}: P_{h} \rightarrow$ $B_{h}$. We assume that $P_{h}$ is convex for every zone $B_{h} \subset$
$\mathcal{B}$; it may be empty for some $B_{h}$. We write $\left|P_{h}\right|$ for the length of the curve $\Gamma_{h}$. The quantization of the length of $\Gamma_{h}$, with quantization constant $\sigma>0$, is defined as $Q_{\sigma}\left(\left|P_{h}\right|\right)=$ $\left\lceil\frac{\left|P_{h}\right|}{\sigma}\right\rceil \sigma$. For the remainder of the paper we fix $\sigma$ and write $Q_{\sigma}\left(\left|P_{h}\right|\right)$ as $Q_{h}$. We also write $Q_{\min }$ and $Q_{\max }$ for the minimum and maximum $Q_{h}$, such that $P_{h} \neq \emptyset$.

Our goal is to design an algorithm that runs on the physical nodes such that, if there are no failures or recoveries after a certain point in time, then: (1) within finite time the set of nodes in each zone $B_{h}, h \in \mathcal{H}$, becomes fixed, and the size of this set is "approximately" proportional to the quantized length $Q_{h}$, (2) within finite time all physical nodes in $B_{h}$ for which $Q_{h} \neq 0$ are located on $\Gamma_{h}$, and (3) in the limit all the nodes in each $B_{h}$ are evenly spaced on $\Gamma_{h}$.

\section{Solution Using Virtual Node LAyer}

In our algorithm each virtual node $V N_{h}, h \in \mathcal{H}$, uses only information about the portions of the target curve $\Gamma$ in zone $B_{h}$ and the neighboring zones. For convenience, we assume that all client nodes know the complete curve $\Gamma$; we could instead model the client nodes in $B_{h}$ as receiving inputs from another automaton about the nature of the curve in zone $B_{h}$ and neighboring zones only.

The Virtual Node abstraction is used as a means to coordinate the movement of client nodes in a zone. A $V N$ controls the motion of the $C N \mathrm{~s}$ in its zone by setting and broadcasting target waypoints for the $C N \mathrm{~s}$ : $V N_{h}$ periodically receives information from clients in its zone, exchanges information with its neighbors, and sends out a message containing a calculated target point for each client node "assigned" to zone $h$. Informally, $V N_{h}$ performs two tasks when setting the target points: (1) it re-assigns some of the $C N$ s that are assigned to itself to neighboring $V N \mathrm{~s}$, and (2) it sends a target position on $\Gamma$ to each $C N$ that is assigned to itself. The objective of (1) is to prevent neighboring $V N \mathrm{~s}$ from getting depleted of $C N \mathrm{~s}$ and to achieve a distribution of $C N \mathrm{~s}$ over the zones that is proportional to the length of $\Gamma$ in each zone. The objective of (2) is to space the nodes evenly on $\Gamma$ in each zone. A $C N$, in turn, receives its current position information from $R W$ and its target location from a $V N$, and continuously computes a velocity vector that will take it to its latest received target point.

\section{A. Client Node Algorithm}

The $C N(\delta)_{i}, i \in \mathcal{I}$, algorithm (see Figure 5) follows a round structure, where rounds begin at times that are multiples of $\delta$. Recall that $V N$ automata do not have access to realtime whereas $C N$ automata do. To help $V N$ s follow the round structure, the $C N \mathrm{~s}$ send "trigger" messages to prompt the $V N$ s to perform transitions.

At the beginning of each round, a $C N$ sends a cn-update message. The cn-update message tells the local $V N$ (in whose zone the $C N$ currently resides) the $C N$ 's $i d$, assigned $V N$, current location in $\mathcal{B}$, and current round number. 


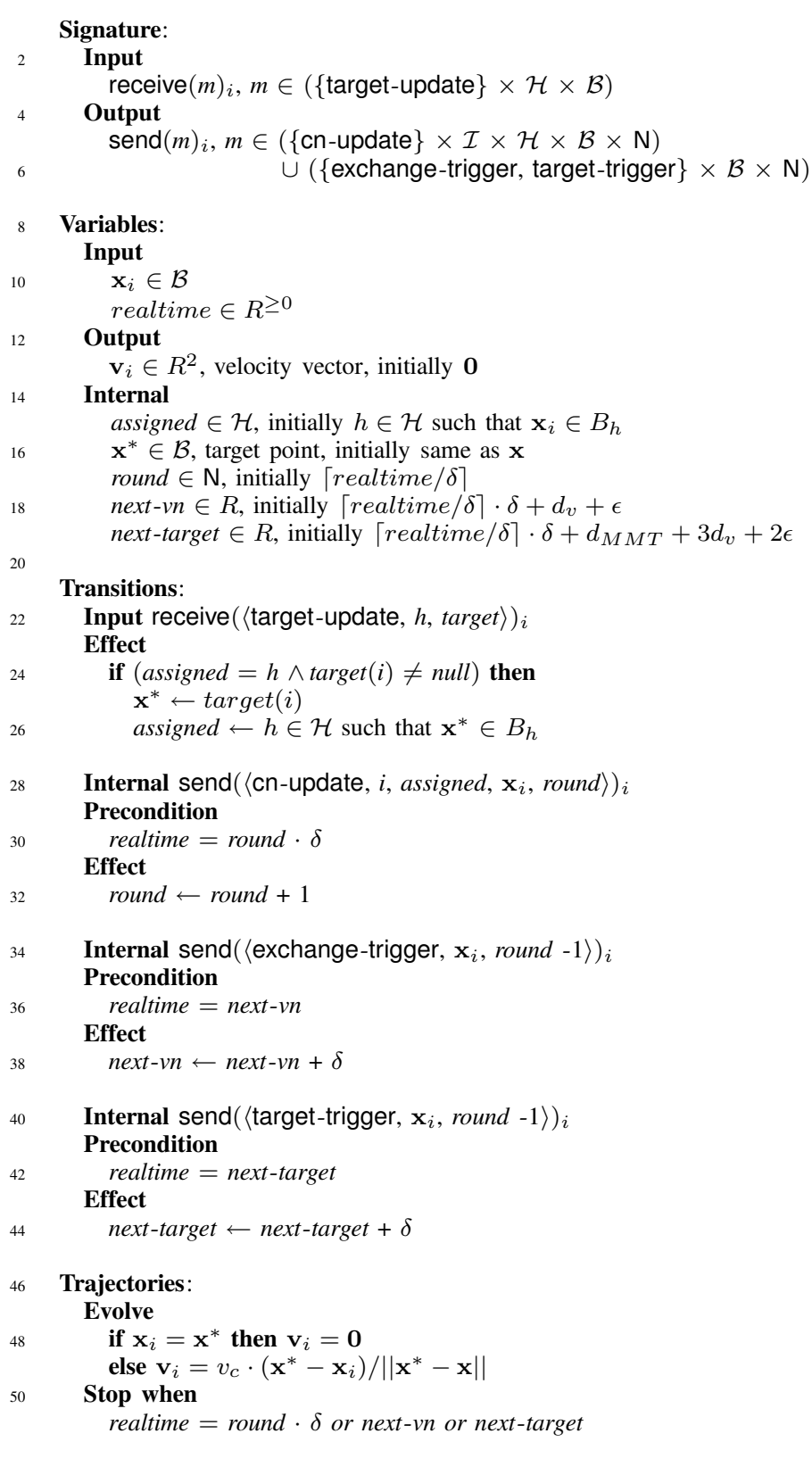

Fig. 5. Client node $C N(\delta)_{i}$ automaton.

The $C N$ then sends an exchange-trigger message $d_{v}+\epsilon$ later to its local $V N$. An additional $d_{M M T}+2 d_{v}+\epsilon$ time later, the $C N$ sends a target-trigger message to its local $V N$. Both these messages are trigger messages that include the $C N$ 's current location and the current round number, used by the local $V N$ to determine whether the $C N$ is in its zone and what the current round number is.

$C N_{i}$ processes only one kind of message, target-update messages sent by its assigned $V N$ (to which it is currently assigned). Each such message describes the new target location $\mathbf{x}_{i}^{*}$ for $C N_{i}$, and possibly an assignment to a different $V N . C N_{i}$ continuously computes its velocity vector $\mathbf{v}_{i}$, based on its current position $\mathbf{x}_{i}$ and its target position $\mathbf{x}_{i}^{*}$, as $\mathbf{v}_{i}=v_{c}\left(\mathbf{x}_{i}-\mathbf{x}_{i}^{*}\right) /\left\|\mathbf{x}_{i}-\mathbf{x}_{i}^{*}\right\|$, moving it with maximum velocity towards the target.

\section{B. Virtual Node Algorithm}

In designing the motion coordination algorithm we make use of the apparent synchrony created by the virtual layer implementation. The $V N\left(e, \rho_{1}, \rho_{2}\right)_{h}, h \in \mathcal{H}$, algorithm (see Figure 6) follows the $C N \mathrm{~s}$ ' round structure. However, $V N$ s do not have access to the realtime variable and must instead rely on trigger messages from $C N$ s to determine when enough time has elapsed to perform required actions. We begin by explaining how we implement the round structure for a $V N$ and then explain the $V N$ algorithm.

Round structure. At the beginning of a round, each $C N$ sends a cn-update message to its local $V N$. The $C N$ s then send exchange-trigger messages $d_{v}+\epsilon$ after the beginning of the round, signalling that the $V N$ has received all $\mathrm{cn}-$ update messages that were transmitted at the beginning of the round in its zone. The $V N$ waits before using information from the cn-update messages until it receives one of the $C N$ s' exchange-trigger messages. The $V N$ then sends vn-update messages to its neighbors.

Each $C N$ sends a target-trigger message to its local $V N d_{M M T}+2 d_{v}+\epsilon$ time after it sends an exchangetrigger message. This is late enough in the round that: (1) neighboring $V N$ s have received an exchange-trigger message ( $d_{v}$ time), (2) each neighboring $V N$ has performed a vn-update transmission to its neighboring $V N \mathrm{~s}$, including this one ( $d_{M M T}$ time), and (3) the neighboring $V N$ transmissions have arrived ( $d_{v}$ time). When a $V N$ first receives a target-trigger message for a particular round from any $C N$ in its region, it knows it has received any vn-update messages from neighboring $V N$ s for the round. The $V N$ then performs some computation and transmits a target-update message to $C N$ s local to it.

A target-update message might not be received by a $C N$ until $d_{M M T}+2 d_{v}$ time after the $C N$ sent the targettrigger message. This accounts for: (1) the time it can take for the target-trigger message to be received by the $V N$ $\left(d_{v}\right)$, (2) the time it can take for the $V N$ to perform the target-update broadcast $\left(d_{M M T}\right)$, and (3) the time for the broadcast to be delivered at the $C N\left(d_{v}\right)$. Given the maximum distance between a point in one zone and the center of a neighboring zone, $\sqrt{2.5} b=\sqrt{(3 b / 2)^{2}+(b / 2)^{2}}$, and a constant speed of $v_{c}$ for each client node, it can take up to $\frac{\sqrt{2.5} b}{v_{c}}$ time for the $C N$ to reach its target. Also, after the $C N$ just arrives in the zone it was assigned to, up to $\sqrt{10} b / 3=\sqrt{2.5} b \cdot \frac{2}{3}$ distance from where it started, it could find that the local $V N$ is failed, in which case it could take up to the $d_{r} V N$-startup time for the $V N$ to recover.

To ensure a round is long enough for a client node to send the cn-update, exchange-trigger, and target-trigger messages, receive a target-update message, arrive at its new assigned target location, and be sure a virtual node is alive in 
its zone before a new round begins, we require that $\delta$ satisfy $\delta>2 d_{M M T}+5 d_{v}+2 \epsilon+\max \left(\sqrt{2.5} b / v_{c}, \sqrt{10} b / 3 v_{c}+d_{r}\right)$.

$V N$ algorithm. Each $V N_{h}$ automaton collects cn-update messages sent at the beginning of the round from $C N \mathrm{~s}$ located in its zone, aggregating the location and round information from the message in a table, $M$. When $V N_{h}$ first receives an exchange-trigger message for a particular round from any $C N$ in its zone, $V N_{h}$ tallies and computes from its table $M$ the number of client nodes assigned to it that it has heard from in the round, and sends this information in a vn-update message to all of its neighbors.

When $\mathrm{VH}_{h}$ receives a vn-update message from a neighboring $V N$, it stores the $C N$ population and round number information from the message in a table, $V$. When $V N_{h}$ first receives a target-trigger message for a particular round from any $C N$ in its region, $V N_{h}$ uses the information in its tables $M$ and $V$ about the number of $C N \mathrm{~s}$ in its zone and its neighbors' zones to calculate how many of the $C N$ s assigned to itself should be reassigned and to which neighboring $V N \mathrm{~s}$. This is done through the assign function (see Figure 7) which calculates a partial function assign mapping $C N$ identifiers to zones that they are assigned to. If the number of $C N \mathrm{~s} y(h)$ assigned to $V N_{h}$ exceeds the minimum critical number $e$, then the assign function reassigns some of the $C N \mathrm{~s}$ to neighbors of $V N_{h}$.

Let $I n_{h}$ denote the set of neighboring $V N$ s of $V N_{h}$ that are on the curve $\Gamma$ and $y_{h}(g), g \in N b r s_{h} \cup\{h\}$, denote the number $\operatorname{num}\left(V_{h}(g)\right)$ of $C N$ s assigned to $V N_{g}$. If $Q_{h} \neq 0$, meaning $V N_{h}$ is on the curve (lines 7-11), then we let lower $_{h}$ denote the subset of $N b r s_{h}$ that are on the curve and have fewer assigned $C N \mathrm{~s}$ than $V N_{h}$ has after normalizing with $\frac{Q_{g}}{Q_{h}}$. For each $g \in$ lower $_{h}, V N_{h}$ reassigns either $r a=$ $\rho_{2} \cdot\left[\frac{Q_{g}}{Q_{h}} y_{h}(h)-y_{h}(g)\right] / 2\left(\mid\right.$ lower $\left._{h} \mid+1\right)$ or the number of nodes over $e$ it has not already reassigned, whichever is smaller, of the $C N$ s that are currently assigned to itself to $V N_{g}$, where $\rho_{2}<1$ is a damping factor.

If $Q_{h}=0$, meaning $V N_{h}$ is not on the curve, and $V N_{h}$ has no neighbors on the curve (lines 13-17), then we let lower $_{h}$ denote the subset of $\mathrm{Nbrs}_{h}$ that have fewer assigned $C N$ s than $V N_{h}$. For each $g \in$ lower $_{h}, V N_{h}$ reassigns either $r a=\rho_{2} \cdot\left[y_{h}(h)-y_{h}(g)\right] / 2\left(\mid\right.$ lower $\left._{h} \mid+1\right)$ or the number of nodes over $e$ it has not already reassigned, whichever is smaller, of the $C N$ s currently assigned to itself to $V N_{g}$.

$V N_{h}$ is on a boundary if $Q_{h}=0$, but there is a $g \in$ $N_{b r s}$ with $Q_{g} \neq 0$. In this case, $y_{h}(h)-e$ of $V N_{h}$ 's $C N \mathrm{~s}$ are assigned equally to neighbors in $\mathrm{In}_{h}$ (lines 1922).

The client assignments are then used to calculate new target points for local $C N$ s through the calctarget function (see Figure 7). This function assigns to every $C N_{i}$ assigned to $V N_{h}$ a target point $l o c M_{h}(i) \in B_{g}, g \in N b r s_{h} \cup\{h\}$, to move to. The target point $\operatorname{loc}_{h}(i)$ is computed as follows: If $C N_{i}$ is assigned to $V N_{g}, g \neq h$, then its target is set to the center $\mathbf{o}_{g}$ of $B_{g}$ (lines 30-31); if $C N_{i}$ is assigned to

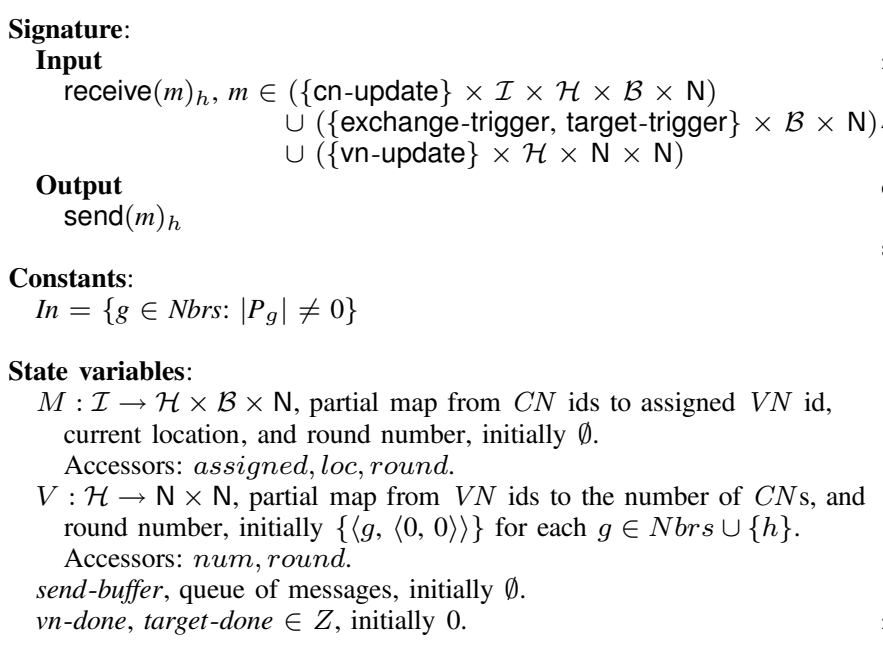

Derived variables:

assigned $M=\{i \in i d(M):$ assigned $(M(i))=h\}$

$\operatorname{loc} M=\lambda(i \in i d(M)) \cdot \operatorname{loc}(M(i))$

$y=\lambda(g: N b r s \cup\{h\})$. num $(V(g))$

Transitions:

Input receive $(\langle\text { cn-update, } i d \text {, assigned, loc, round }\rangle)_{h}$

Effect

if $l o c \in B_{h}$ then

$M \leftarrow M \cup\{\langle i d,\langle$ assigned, loc, round $\rangle\rangle\}$

Input receive $(\langle\text { exchange-trigger, loc, round }\rangle)_{h}$ Effect

if $\left(\right.$ loc $\in B_{h} \wedge v n$-done $\neq$ round $)$ then

for each $i \in i d(M)$

if $\operatorname{round}(M(i)) \neq$ round then

$M \leftarrow M \backslash\{\langle i, M(i)\rangle\}$

$V(h) \leftarrow\langle|$ assigned $M \mid$, round $\rangle$

send-buffer $\leftarrow$ send-buffer $\cup\{\langle\mathrm{vn}$-update, $h, y(h)$, round $\rangle\}$

vn-done $\leftarrow$ round

Input receive $(\langle\mathrm{vn} \text {-update, } i d, n, \text { round }\rangle)_{h}$

Effect

if $i d \in N b r s$ then

$V(i d) \leftarrow\langle n$, round $\rangle$

$$
10
$$

2

4

$$
16
$$

18

20

24

26

28

30

Input receive $(\langle\text { target-trigger, loc, round }\rangle)_{h}$ Effect

if $\left(\right.$ loc $\in B_{h} \wedge$ target-done $\neq$ round) then

for each $i \in i d(M)$

if $\operatorname{round}(M(i)) \neq$ round then

$M \leftarrow M \backslash\{\langle i, M(i)\rangle\}$

$V(h) \leftarrow\langle|$ assigned $M \mid$, round $\rangle$

for each $g \in$ Nbrs

if $\operatorname{round}(V(g)) \neq$ round then $V(g) \leftarrow\langle 0,0\rangle$

let target $=$ calctarget $($ assign $($ assigned $M, y)$, locM $)$

send-buffer $\leftarrow$ send-buffer $\cup\{\langle$ target-update, $h$, target $\rangle\}$

target-done $\leftarrow$ round

Output send $(m)_{i}$

Precondition

send-buffer $\neq \emptyset \wedge m=\operatorname{head}($ send-buffer $)$

Effect

send-buffer $\leftarrow$ tail (send-buffer $)$

Tasks and bounds:

$\left\{\operatorname{send}(m)_{h}\right\}$, bounds $\left[0, d_{M M T}\right]$

Fig. 6. $V N\left(e, \rho_{1}, \rho_{2}\right)_{h}$ IOA signature, variables, transitions, and tasks, implementing motion coordination algorithm with parameters: safety $e$, and damping $\rho_{1}, \rho_{2}$. 


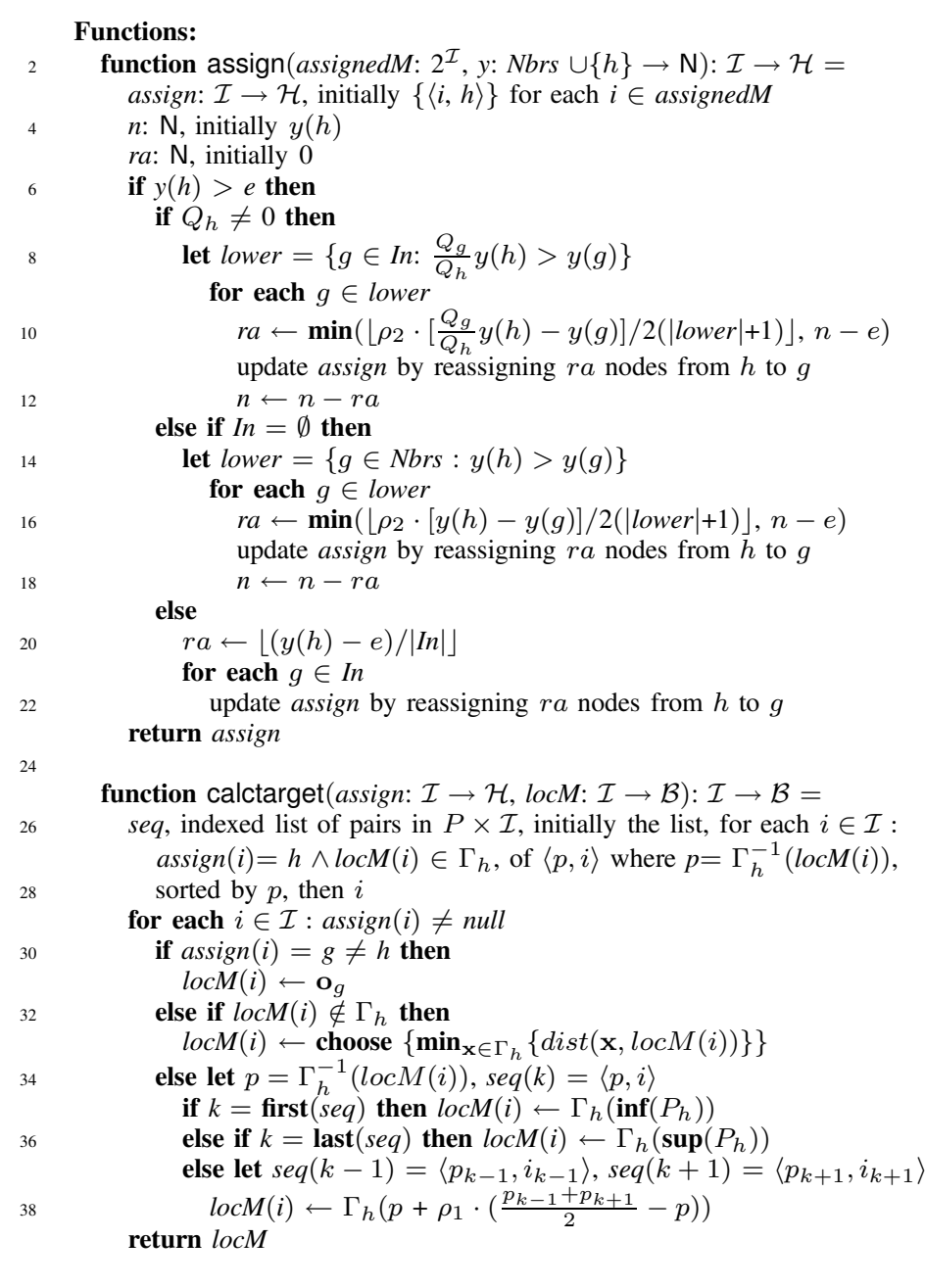

Fig. 7. $V N\left(e, \rho_{1}, \rho_{2}\right)_{h}$ IOA functions.

$V N_{h}$ but is not located on the curve $\Gamma_{h}$ then its target is set to the nearest point on the curve, nondeterministically choosing one if there are several (lines 32-33); if $C N_{i}$ is either the first or last client node on $\Gamma_{h}$ then its target is set to the corresponding endpoint of $\Gamma_{h}$ (lines 35-36); if $C N_{i}$ is on the curve but is not the first or last client node then its target is moved to the mid-point of the locations of the preceding and succeeding $C N$ s on the curve (line 38). For the last two computations a sequence $s e q_{h}$ of nodes on the curve sorted by curve location is used (line 27).

$V N_{h}$ finally broadcasts the new target waypoints for the round through a target-update message to its $C N \mathrm{~s}$.

\section{Vi. Correctness and Performance}

We say $C N_{i}, i \in \mathcal{I}$, is active in round $t$ if its mode is active for the duration of round $t$. A $V N_{h}, h \in \mathcal{H}$, is active in round $t$ if there is some active $C N_{i}$ with $\mathbf{x}_{i} \in B_{h}$ for the duration of rounds $t-1$ and $t$. None of the $V N \mathrm{~s}$ are active in the starting round 0 . We use the following notation: $\operatorname{In}(t)$ is the set of ids $h \in \mathcal{H}$ of $V N$ s that are active in round $t$ and for which $Q_{h} \neq 0$. Out $(t)$ is the set of ids $h \in \mathcal{H}$ of
$V N$ s that are active in round $t$ and for which $Q_{h}=0 . C(t)$ is the set of active $C N \mathrm{~s}$ at round $t$, and $C_{\text {in }}(t)$ and $C_{\text {out }}(t)$ are the sets of active $C N$ s located in zones in $\operatorname{In}(t)$ and Out $(t)$, respectively, at the beginning of round $t$.

For any pair of neighboring zones $g$ and $h$, and for any round $t$, we use $y_{g}(h)(t)$ to refer to the value of $y_{g}(h)$ at the point in time in round $t$ when $V N_{g}$ finishes processing the first target-trigger message of round $t$. For any $f, g \in$ $N b r s_{h} \cup\{h\}$, in the absence of failures and recoveries of $C N \mathrm{~s}$ in round $t, y_{f}(h)(t)=y_{g}(h)(t)$; we write this simply as $y_{h}(t)$.

In the following subsection we prove that the $V N$ algorithm satisfies our first goal, that is, if there are no failures or recoveries of $C N \mathrm{~s}$ after a certain round $t_{0}$, then within a finite number of rounds after $t_{0}$, a round $T_{s t a b}$ is reached after which: (1) the set of $C N \mathrm{~s}$ assigned to each $V N$ is fixed, and (2) the number of $C N$ s assigned to each $V N_{h}$ such that $Q_{h} \neq 0$ is proportional to $Q_{h}$ within a constant additive factor.

\section{A. Assignments Stabilize}

For each of the following lemmas, we assume that there are no failures or recoveries of $C N \mathrm{~s}$ after round $t_{0}$. The first lemma states some basic facts about the assign function (see Figure 7):

Lemma 1: In every round $t>t_{0}$ : (1) $\operatorname{In}(t) \subseteq \operatorname{In}(t+1)$, (2) $O u t(t) \subseteq O u t(t+1)$, (3) $C_{\text {in }}(t) \subseteq C_{\text {in }}(t+1)$, (4) $C_{\text {out }}(t+1) \subseteq C_{\text {out }}(t)$, and (5) if $y_{h}(t) \geq e$ for some $h \in \mathcal{H}$, then $y_{h}(t+1) \geq e$.

The next lemma states a key property of the assign function after round $t_{0}: V N_{h}, h \in O u t(t)$, is never assigned a larger number of $C N \mathrm{~s}$ in round $t+1$ than the largest number of $C N \mathrm{~s}$ that were assigned to any of $V N_{h}$ 's neighbors in round $t$. A similar property holds for $V N_{h}$, $h \in \operatorname{In}(t)$, with respect to the density of $C N \mathrm{~s}$.

Lemma 2: In every round $t>t_{0}$, for $g, h \in \mathcal{H}$ with $h \in N b r s_{g}$ :

(1) If $g, h \in \operatorname{Out}(t), y_{h}(t)=\max _{f \in N b r s_{g}} y_{f}(t)$, and $y_{g}(t)<y_{h}(t)$, then $y_{g}(t+1) \leq y_{h}(t)-1$, and

(2) If $g, h \in \operatorname{In}(t), \frac{y_{h}(t)}{Q_{h}}=\max _{f \in N b r s_{g}} \frac{y_{f}(t)}{Q_{f}}$, and $\frac{y_{g}(t)}{Q_{g}}<$ $\frac{y_{h}(t)}{Q_{h}}$, then $\frac{y_{g}(t+1)}{Q_{g}} \leq \frac{y_{h}(t)}{Q_{h}}-\frac{\sigma}{Q_{\text {max }}^{2}}$.

Proof: (1) Fix $g, h$ and $t$, as in the statement of the lemma. Since $y_{h}(t)>y_{g}(t)$ and $g, h \in \operatorname{Out}(t)$, we see from line 16 of Figure 7 that the number of $C N$ s that $V N_{g}$ is assigned from $V N_{h}$ in round $t$ is at most $\rho_{2}\left(y_{h}(t)-y_{g}(t)\right) / 2\left(\mid\right.$ lower $\left._{h}(t) \mid+1\right)$. This is at most $\rho_{2}\left(y_{h}(t)-y_{g}(t)\right) / 4$, because $y_{h}(t)>y_{g}(t)$ implies that lower $_{h}(t) \geq 1$. Then, the total number of $C N$ s assigned to $V N_{g}$ in round $t$ by all four of its neighbors is at most $\rho_{2}\left(y_{h}(t)-y_{g}(t)\right)$. Therefore, $y_{g}(t+1) \leq y_{g}(t)+\rho_{2}\left(y_{h}(t)-\right.$ $\left.y_{g}(t)\right)=\rho_{2} y_{h}(t)+\left(1-\rho_{2}\right) y_{g}(t)$. As $\rho_{2}<1$, we have $y_{g}(t+1)<y_{h}(t)$. The result follows from integrality of $y_{g}(t+1)$ and $y_{h}(t)$. 
(2) As in part 1, fix $g, h$ and $t$. Here $\frac{y_{h}(t)}{Q_{h}}>\frac{y_{g}(t)}{Q_{g}}$ and $g, h \in \operatorname{In}(t)$. From line 10 of Figure 7, it follows that the number of $C N \mathrm{~s}$ that $V N_{g}$ is assigned from $V N_{h}$ in round $t$ is at most $\rho_{2}\left(\frac{Q_{g}}{Q_{h}} y_{h}(t)-y_{g}(t)\right) / 2\left(\mid\right.$ lower $_{h}(t) \mid+$ $1)$. This is at most $\rho_{2}\left(\frac{Q_{g}}{Q_{h}} y_{h}(t)-y_{g}(t)\right) / 4$. Then, the total number of $C N \mathrm{~s}$ assigned to $V N_{g}$ in round $t$ by all four of its neighbors is at most $\rho_{2}\left(\frac{Q_{g}}{Q_{h}} y_{h}(t)-y_{g}(t)\right)$. Therefore, $y_{g}(t+1) \leq\left(1-\rho_{2}\right) y_{g}(t)+\rho_{2} \frac{Q_{g}}{Q_{h}} y_{h}(t)$, that is $\frac{y_{g}(t+1)}{Q_{g}} \leq$ $\left(1-\rho_{2}\right) \frac{y_{g}(t)}{Q_{g}}+\rho_{2} \frac{y_{h}(t)}{Q_{h}}$. As $\rho_{2}<1$, we have $\frac{y_{g}(t+1)}{Q_{g}}<$ $\frac{y_{h}(t)}{Q_{h}}$. A simple calculation shows that if $\frac{y_{h}(t)}{Q_{h}} \neq \frac{y_{g}(t)}{Q_{g}}$, then $\frac{y_{h}(t)}{Q_{h}}-\frac{y_{g}(t)}{Q_{g}} \geq \frac{\sigma}{Q_{\max }^{2}}$.

Lemma 3: There exists a round $T_{i n}>t_{0}$ such that in any round $t \geq T_{i n}$, the number of $C N \mathrm{~s}$ assigned to $V N_{h}$, $h \in O u t(t)$, is unchanged: $y_{h}(t+1)=y_{h}(t)$.

Proof: Let $N_{\text {out }}$ be the total number of $h \in \mathcal{H}$ such that $Q_{h}=0$. For any $k, 1 \leq k \leq N_{\text {out }}$, we define $\max _{k}(t)$ to be the $k^{t h}$ largest number of $C N \mathrm{~s}$ that are assigned to any $V N_{h}, h \in O u t(t)$, at the beginning of round $t>t_{0}$ :

$\max _{k}(t) \triangleq\left\{\begin{aligned} \max \left\{y_{h}(t): h \in O u t(t)\right\}, & \text { if } k=1 \\ \max \left\{y_{h}(t): h \in O u t(t) \wedge\right. & \\ \left.y_{h}(t)<\max _{k-1}(t)\right\}, & \text { otherwise. }\end{aligned}\right.$

Let $\operatorname{maxvns}_{k}(t)$ be the set of $V N$ ids that have $\max _{k}(t)$ $C N$ s assigned to them. If there exists an $l, 1 \leq l \leq N_{\text {out }}$, such that $\forall h \in O u t(t): \max _{l}(t) \geq y_{h}(t)$, then for all $k$, $l<k \leq N_{\text {out }}, \max _{k}(t)=0$ and $\operatorname{maxvns}_{k}(t)=\emptyset$.

Let $E(t)=\left(\left|C_{\text {out }}(t)\right|, \max _{1}(t),\left|\operatorname{maxvns}_{1}(t)\right|, \ldots\right.$, $\left.\max _{N_{\text {out }}}(t),\left|\operatorname{maxvn}_{N_{\text {out }}}(t)\right|\right)$. Let $w$ be the minimum $y_{h}\left(t_{0}\right)$ for any $h \in O u t\left(t_{0}\right)$, and $S=\left\{h \in O u t\left(t_{0}\right)\right.$ : $\left.y_{h}\left(t_{0}\right)=w\right\}$. Observe that if $w<e$, then $E_{\text {min }}=$ $(w|S|, w,|S|, 0,0 \ldots, 0,0)$ is a minimum value for $E(t)$, otherwise $E_{\text {min }}=(e|S|, e,|S|, 0,0 \ldots, 0,0)$ is a minimum value. It suffices to show that for any round $t>t_{0}$, either $E(t+1)=E(t)$, that is, $t=T_{i n}$, or $E(t+1)$ is less than $E(t)$ by some constant amount, meaning there is a $k, 1 \leq k \leq N_{\text {out }}$, such that for every $l, 1 \leq l<k$, the $l^{t h}$ component of $E(t+1)$ is equal to the $l^{t h}$ component of $E(t)$, and the $k^{t h}$ component of $E(t+1)$ is less than the $k^{t h}$ component of $E(t)$ by at least 1 .

Consider any round $t$ after $t_{0}$. From Lemma 1 we know that $\left|C_{\text {out }}(t+1)\right| \leq\left|C_{\text {out }}(t)\right|$. If $\left|C_{\text {out }}(t+1)\right|<\left|C_{\text {out }}(t)\right|$, then the first component of $E(t+1)$ is less than that of $E(t)$ by at least 1 . Otherwise, $\left|C_{\text {out }}(t+1)\right|=\left|C_{\text {out }}(t)\right|$. If for every $h \in O u t(t), r a=0$ for all $g \in \operatorname{lower}_{h}(t)$ (see line 16 of Figure 7), then none of the $C N \mathrm{~s}$ in $C_{\text {out }}(t)$ are reassigned in round $t+1$, and $E(t+1)=E(t)$. Setting $T_{i n}=t$, we are done. Otherwise, there exists a nonempty set of $V N \mathrm{~s}$ with ids in $O u t(t)$ that reassign some $C N \mathrm{~s}$ to a neighboring $V N$. We select the nonempty set $A$ of such $V N \mathrm{~s}$ with the highest number of assigned $C N \mathrm{~s}$. Let $A \subseteq \operatorname{maxvns}_{k}(t)$, for some $k, 1 \leq k \leq N_{\text {out }}$.

For any $g \in O u t(t)$ with $y_{g}(t)<\max _{k}(t)$, the maximum value of $y_{h}(t)$ for any $h \in N b r s_{g}$ such that $V N_{g}$ gets some $C N$ s from $V N_{h}$ in round $t$ is at most $\max _{k}(t)$. From Part(1) of Lemma 2 it follows that $y_{g}(t+1) \leq \max _{k}(t)-1$.

For any $V N_{h}, h \in A$, since no $V N$ with $y>$ $\max _{k}(t)$ assigns any $C N$ s to $V N_{h}, y_{h}(t+1)=y_{h}(t)-$ $\sum_{g \in \text { lower }_{h}(t)} r a_{g}(t)$, where $r a_{g}$ is the number of $C N \mathrm{~s}$ $V N_{h}$ assigns to its neighbor $V N_{g}$ in round $t$. We have shown above that for any $g \in O u t(t)$, if $y_{g}(t)<\max _{k}(t)$ then $y_{g}(t+1) \leq \max _{k}(t)-1$. There are two possible cases: (1) if maxvns $_{k}(t)=A$, then the $k^{\text {th }} \max$ decreases, $\max _{k}(t+1) \leq \max _{k}(t)-1$. That is, the $(2 k+1)^{s t}$ component of $E$ decreases by at least 1 , and (2) if $A \subset \operatorname{maxvns}_{k}(t)$, then $\max _{k}(t+1)=\max _{k}(t)$ and $\mid$ maxvns $_{k}(t+1)|=| \operatorname{maxvns}_{k}(t)|-| A \mid$. That is, the $(2 k+2)^{n d}$ component of $E$ decreases by at least 1 .

Corollary 1: In every round $t \geq T_{\text {in }}$, the set of $C N \mathrm{~s}$ assigned to $V N_{h}, h \in O u t(t)$, is unchanged.

Proof: Suppose the set of $C N \mathrm{~s}$ assigned to $V N_{h}$ changes in some round $t \geq T_{i n}$. We know that $y_{h}(t+1)=$ $y_{h}(t)$ for all $h \in O u t(t)$. Summing, $\left|C_{\text {out }}(t+1)\right|=$ $\left|C_{\text {out }}(t)\right|$ and using Lemma 1 we get $C_{\text {out }}(t+1)=C_{\text {out }}(t)$. The only way the set of $C N \mathrm{~s}$ assigned to $V N_{h}$ could change, without changing $y_{h}$ and the set $C_{\text {out }}$, is if there existed a cyclic sequence of $V N \mathrm{~s}$ with ids in $O u t(t)$ in which each $V N$ gives up $c>0 C N$ s to its successor $V N$ in the sequence, and receives $c C N$ s from its predecessor. However, such a cycle of $V N$ s cannot exist because the lower set imposes a strict partial ordering on the VNs.

Corollary 1 implies that in every round $t \geq T_{i n}, \operatorname{In}(t)=$ $\operatorname{In}\left(T_{i n}\right), C_{i n}(t)=C_{\text {in }}\left(T_{i n}\right)$, and $C_{\text {out }}(t)=C_{\text {out }}\left(T_{\text {in }}\right)$; we denote these simply as $I n, C_{i n}$, and $C_{\text {out }}$.

Corollary 2: $\left|C_{\text {out }}\right|=O\left(\mathrm{~m}^{3}\right)$.

Proof: From Corollary 1, the set of $C N \mathrm{~s}$ assigned to each $V N_{h}, h \in O u t(t)$, is unchanged in every round $t \geq T_{i n}$. This implies that in any round $t \geq T_{i n}$, the number of $C N \mathrm{~s}$ assigned by $V N_{h}$ to any of its neighbors is 0 . Therefore, from line 20 of Figure 7, for any boundary $V N_{g},\left(y_{g}(t)-e\right) /\left|I n_{g}\right|<1$. In $n_{g}$ is the (constant) set of $h \in$ Nbrs $_{g}$ with $Q_{h} \neq 0$. Since $\left|I n_{g}\right| \leq 4, y_{g}(t)<4+e$. From line 16 of Figure 7, for any non-boundary $V N_{g}$, $g \in O u t(t)$, that is 1-hop away from a boundary $V N_{h}$, $\frac{\rho_{2}\left(y_{g}(t)-y_{h}(t)\right)}{2\left(\mid \text { lower }_{g}(t) \mid+1\right)}<1$. Since $\mid$ lower $_{g}(t) \mid \leq 4, y_{g}(t) \leq$ $\frac{10}{\rho_{2}}+4+e$. Inducting on the number of hops, the maximum
. number of $C N \mathrm{~s}$ assigned to a $V N_{g}, g \in O u t(t)$, at $l$ hops from the boundary is at most $\frac{10 l}{\rho_{2}}+e+4$. Since for any $l, 1 \leq l \leq 2 m-1$, there can be at most $m$ $V N$ s at $l$-hop distance from the boundary, summing gives $\left|C_{\text {out }}\right| \leq(e+4)(2 m-1) m+\frac{10 m^{2}(2 m-1)}{\rho_{2}}=O\left(m^{3}\right)$.

Lemma 4: There exists a round $T_{\text {stab }} \geq T_{i n}$ such that in every round $t \geq T_{s t a b}$, the set of $C N$ s assigned to $V N_{h}$, $h \in I n$, is unchanged.

The next lemma states that the number of $C N$ s assigned to each $V N_{h}, h \in I n$, in the stable assignment after $T_{s t a b}$ is proportional to $Q_{h}$ within a constant additive factor. 
Lemma 5: In every round $t \geq T_{\text {stab }}$, for $g, h \in \operatorname{In}(t)$ :

$$
\left|\frac{y_{h}(t)}{Q_{h}}-\frac{y_{g}(t)}{Q_{g}}\right| \leq\left[\frac{10(2 m-1)}{Q_{\min } \rho_{2}}\right] .
$$

Proof: Consider a pair of $V N \mathrm{~s}$ for neighboring zones $B_{g}$ and $B_{h}, g, h \in I n$. Assume w.l.o.g. $y_{h}(t) \geq y_{g}(t)$. From line 10 of Figure 7, it follows that $\rho_{2}\left(\frac{Q_{g}}{Q_{h}} y_{h}(t)-y_{g}(t)\right) \leq$ $2\left(\left|\operatorname{lower}_{h}(t)\right|+1\right)$. Since $\left|\operatorname{lower}_{h}(t)\right| \leq 4,\left|\frac{y_{h}(t)}{Q_{h}}-\frac{y_{g}(t)}{Q_{g}}\right| \leq$ $\frac{10}{Q_{g} \rho_{2}} \leq \frac{10}{Q_{\text {min }} \rho_{2}}$. By induction on the number of hops from 1 to $2 m-1$ between any two $V N$ s, the result follows.

\section{B. On the Curve and Evenly Spaced}

We continue to assume that there are no failures or recoveries of $C N \mathrm{~s}$ after round $t_{0}$.

From line 33 of Figure 7, it follows immediately that by the beginning of round $T_{s t a b}+2$, all $C N \mathrm{~s}$ in $C_{i n}$ are located on the curve $\Gamma$. This establishes that the $V N$ algorithm satisfies our second goal. In the remainder of this section, we prove that the locations of the $C N \mathrm{~s}$ in each zone $B_{h}$, $h \in I n$, are evenly spaced on $\Gamma_{h}$ in the limit.

Lemma 6: Consider a sequence of rounds $t_{1}=$ $T_{s t a b}, \ldots, t_{n}$. As $n \rightarrow \infty$, the locations of $C N \mathrm{~s}$ in $B_{h}$, $h \in I n$, are evenly spaced on $\Gamma_{h}$.

Proof: From Lemma 4 we know that the set of $C N \mathrm{~s}$ assigned to each $V N_{h}, h \in I n$, remains unchanged. Then, at the beginning of round $t_{2}$, every $C N$ assigned to $V N_{h}$ is located in $B_{h}$ and is on the curve $\Gamma_{h}$. Assume w.l.o.g. that $V N_{h}$ is assigned at least two $C N$ s. Then, at the beginning of round $t_{3}$, one $C N$ is positioned at each endpoint of $\Gamma_{h}$, namely at $\Gamma_{h}\left(\inf \left(P_{h}\right)\right)$ and $\Gamma_{h}\left(\sup \left(P_{h}\right)\right)$. From lines 3536 of Figure 7, we see that the target points for these endpoint $C N \mathrm{~s}$ are not changed in successive rounds. Let $\operatorname{seq}_{h}\left(t_{2}\right)=\left\langle p_{0}, i_{(0)}\right\rangle, \ldots,\left\langle p_{n+1}, i_{(n+1)}\right\rangle$, where $y_{h}=n+2$, $p_{0}=\inf \left(P_{h}\right)$, and $p_{n+1}=\sup \left(P_{h}\right)$. From line 38 of Figure 7, for any $i, 1<i<n$, the $i^{t h}$ element in $s e q_{h}$ at round $t_{k}, k>2$, is given by:

$p_{i}\left(t_{k+1}\right)=p_{i}\left(t_{k}\right)+\rho_{1}\left(\frac{p_{i-1}\left(t_{k}\right)+p_{i+1}\left(t_{k}\right)}{2}-p_{i}\left(t_{k}\right)\right)$.

For the endpoints, $p_{i}\left(t_{k+1}\right)=p_{i}\left(t_{k}\right)$. Let the $i^{t h}$ evenly spaced point on the curve $\Gamma_{h}$ between the two endpoints be $\overline{\mathbf{x}}_{i}$. The parameter value $\bar{p}_{i}$ corresponding to $\overline{\mathbf{x}}_{i}$ is given by $\bar{p}_{i}=p_{0}+\frac{i}{n+1}\left(p_{n+1}-p_{0}\right)$. In what follows, we show that as $n \rightarrow \infty$, the $p_{i}$ converge to $\bar{p}_{i}$ for every $i, 0<i<n+1$, that is, the location of the non-endpoint $C N \mathrm{~s}$ are evenly spaced on $\Gamma_{h}$. [[The rest of this proof is exactly the same as the proof of Theorem 3 in [8]. They prove convergence of points on a straight line with even spacing, which is the same as proving convergence of the parameters in our case. I am writing this here to make the proof complete, but we should just cite their paper.]]

Observe that $\bar{p}_{i}=\frac{1}{2}\left(\bar{p}_{i-1}+\bar{p}_{i+1}\right)=\left(1-\rho_{1}\right) \bar{p}_{i}+$ $\frac{\rho_{1}}{2}\left(\bar{p}_{i-1}+\bar{p}_{i+1}\right)$. Define error at step $k, k>2$, as $e_{i}(k)=$ $p_{i}\left(t_{k}\right)-\bar{p}_{i}$. Therefore, for each $i, 2 \leq i \leq n-1, e_{i}(k+1)=$ $p_{i}\left(t_{k+1}\right)-\bar{p}_{i}=\left(1-\rho_{1}\right) e_{i}(k)+\frac{\rho_{1}}{2}\left(e_{i-1}(k)+e_{i+1}(k)\right)$,

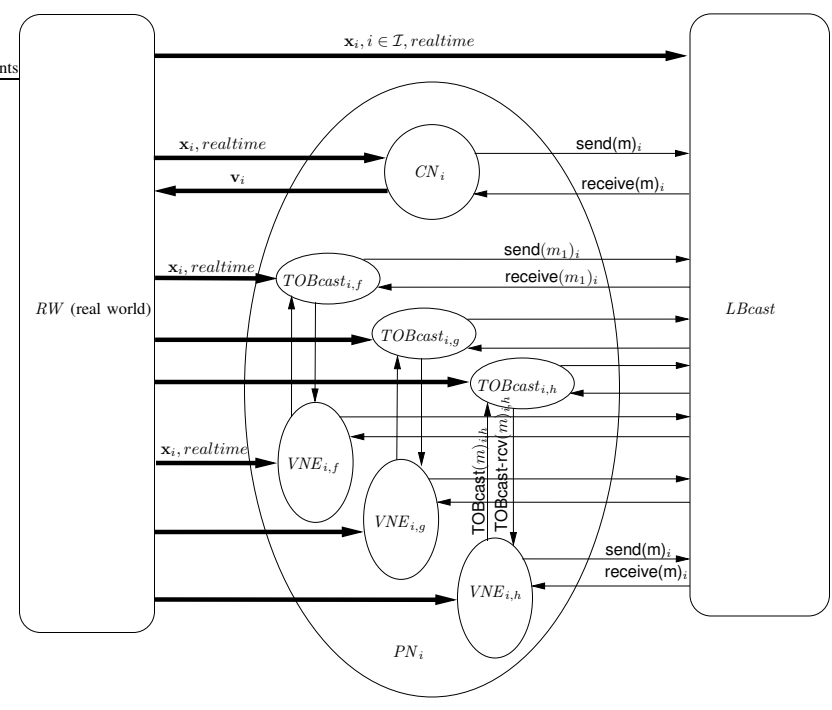

Fig. 8. $P N_{i}$ 's subautomata: A physical node runs several programs, including $V N E$ and TOBcast automata as well as a $C N$ automaton.

$e_{1}(k+1)=\left(1-\rho_{1}\right) e_{1}(k)+\frac{\rho_{1}}{2} e_{2}(k)$, and $e_{n}(k+1)=$ $\left(1-\rho_{1}\right) e_{n}(k)+\frac{\rho_{1}}{2} e_{n-1}(k)$. The matrix for this can be written as: $e(k+1)=T e(k)$, where $T$ is an $n \times n$ matrix:

$$
\left[\begin{array}{cccccc}
1-\rho_{1} & \rho_{1} / 2 & 0 & 0 & \ldots & 0 \\
\rho_{1} / 2 & 1-\rho_{1} & \rho_{1} / 2 & 0 & \cdots & 0 \\
\cdot & \cdot & \cdot & \cdot & \cdot & \cdot \\
0 & \cdots & 0 & \rho_{1} / 2 & 1-\rho_{1} & \rho_{1} / 2 \\
0 & \cdots & 0 & 0 & 1-\rho_{1} & \rho_{1} / 2
\end{array}\right] .
$$

Using symmetry of $T, \rho_{1} \leq 1$, and some standard theorems from control theory, it follows that the largest eigenvalue of $T$ is less than 1 . This implies $\lim _{k \rightarrow \infty} T^{k}=0$, which implies $\lim _{k \rightarrow \infty} e(k)=0$.

\section{IMPLEMENTING THE VIRTUAL NODE LAYER}

In addition to client $C N_{i}$, a physical node $P N_{i}, i \in \mathcal{I}$, in zone $B_{h}$ runs a TOBcast $i, h$ service and a $V N E_{i, h}, h \in \mathcal{H}$, algorithm (see Figure 8) to help implement each virtual node $V N_{h}$ and the $V L B$ cast service of the virtual layer.

In this section we present a sketch of our implementation of the virtual layer by the physical layer. Our implementation is an adaptation of techniques from [6] to emulate a virtual mobile node. The only substantive changes made in our current implementation are: (1) the changing of virtual node locations to be stationary, (2) the replacement of a periodic location update with a continuous real-time location update, and (3) the restart of a virtual node as soon as a physical node discovers it is in a failed virtual node's zone. The virtual nodes we implement here are also modeled differently from those in [6], as MMT automata, rather than simple I/O automata.

We use a standard replicated state machine approach to implement robust virtual nodes that takes advantage of a $T O B$ cast service to ensure that all $V N E \mathrm{~s}$ in a zone receive the same messages in the same order. Using the LBcast 
service of the physical nodes and common knowledge about realtime, the totally ordered broadcast service TOBcast for a zone can be implemented as follows: At the time of sending, a message is tagged with the sender's identifier, zone id, and a timestamp, which is the current value of realtime. Assuming that a $P N$ does not make multiple broadcasts at the same point in time, the tags define a total order on sent messages. Before delivering a message TOB cast $_{i, h}$ waits until $d_{p}+\epsilon$ time has elapsed since it was sent, ensuring that earlier messages were received. TOB cast ${ }_{i, h}$ only processes messages tagged for zone $B_{h}$.

Each $V N E_{i, h}$ independently maintains the state of $V N_{h}$ and simulates performing actions of the $V N$ on that state. In order to keep the state replication consistent across different $V N E$ s running on different physical nodes in the same zone, when $V N E_{i, h}$ wants to simulate an action of the $V N$, it broadcasts a suggestion to perform the action to the other $V N E$ s of the region using the TOBcast service. This action could, for example, be a suggestion to receive a message on behalf of the $V N$ that was actually received by $V N E_{i, h}$. When an action suggestion is received by $V N E_{i, h}$, it is saved in a pending-action queue. Actions are removed from a pending-action queue in order by $V N E_{i, h}$ and simulated on $V N E_{i, h}$ 's local version of the $V N$ state. A completed action is then moved into a completed-action queue, referenced by $V N E_{i, h}$ to prevent reprocessing of completed actions.

When a $V N E$ enters a zone, it executes a join protocol to get the zone's $V N$ state. The join protocol begins by using $T O B$ cast to send a join-req message. Whenever a $V N E$ receives its own join-req message, it starts saving messages to process in its pending-action queue. If a $V N E$ that has already joined receives the join-req, it uses TOBcast to send a join-ack containing a copy of its version of the $V N$ state. When the joining $V N E$ receives the join-ack, it copies the included $V N$ state and starts processing the actions in its pending-action queue. If a $V N E$ 's join-req is not answered in $2 d_{p}+2 \epsilon$ time, indicating the $V N$ is failed, the $V N E$ will reset the $V N \epsilon$ time later by using TOBcast to send a reset message. When a $V N E$ receives a reset message, it sets the $V N$ state to its initial state, clears the pending-action queue, and starts simulating the $V N$.

Theorem 1: Assuming $R_{p} \geq \sqrt{5}$, the TOBcast $t_{i, h}$, $V N E_{i, h}, i \in \mathcal{I}, h \in \mathcal{H}$, and trivial client implementation correctly implement the Virtual Node abstraction with $V N$ task upper time bound $d_{M M T}=d_{p}+\epsilon, V N$-startup time $d_{r}=3 d_{p}+4 \epsilon, V L B$ cast broadcast radius $R_{v} \geq b$, and $V L B$ cast maximum message delay $d_{v}=2 d_{p}+\epsilon$.

Proof: The correctness of the implementation of the Virtual Node layer largely follows from the proof of correctness for the implementation of the VMN layer in [6]. We here discuss the correctness of the implementation with respect to: (1) the task upper bound, (2) the $V N$-startup time, and (3) the requirements for LBcast and VLBcast.

(1) Once one of an abstract $V N_{h}$ 's output or internal transitions is enabled, the precondition for sending a suggestion to simulate the action through TOBcast is satisfied at all $V N E_{i, h}$ for $P N_{i}$ in $B_{h}$, and the broadcast occurs. It takes at most $d_{p}+\epsilon$ time for the message to be delivered at other $V N E_{i, h}$ for $P N_{i}$ in $B_{h}$, after which the action is simulated. Given that $P N$ transitions are assumed to be instantaneous, $d_{M M T}=d_{p}+\epsilon$.

(2) If $P N_{i}$ enters a zone $B_{h}$ with a failed $V N$, its $V N E_{i, h}$ 's join-req will not be answered in $2 d_{p}+2 \epsilon$ time, and the $V N E$ will send a reset message an additional $\epsilon$ later. It takes the $V N E$ at most $d_{p}+\epsilon$ time to receive the reset message and restart the $V N$. The total time $3 d_{p}+4 \epsilon$ for a joining node to succeed in restarting a $V N$ is $d_{r}$.

(3) As in [6], $d_{v}=2 d_{p}+\epsilon$ since the underlying LBcast service used to implement VLBcast takes up to $d_{p}$ time to deliver a transmitted message from a $V N$ or $C N$, after which TOBcast takes an additional $d_{p}+\epsilon$ time to redeliver a message at a receiving $V N$. Also similarly to [6], we require that $R_{p} \geq \sqrt{5} b$, in order to guarantee that $R_{v} \geq b$, allowing a $C N_{i}$ in $B_{h}, i \in \mathcal{I}, h \in \mathcal{H}$, and $V N_{h}$ to communicate, and a $V N_{h}$ (located at $\mathbf{o}_{h}$ ) and each of its neighboring zones' $V N_{g}, g \in N b r s(h)$, (located at $\mathbf{o}_{g}$ ) to communicate. This is because a $V N E$ emulating a zone $B_{h}$ can be as far away as $\sqrt{(2 b)^{2}+b^{2}}$ from a $V N E$ emulating the $V N$ of neighboring zone $B_{g}$. To guarantee the two can communicate while emulating their respective $V N \mathrm{~s}$, the broadcast radius $R_{p}$ of the physical $L$ Bcast service must be be at least $\sqrt{5} b$. Unlike [6], however, we do not require an additional tolerance factor to account for periodic location updates from the $R W$; here, the $R W$ automaton is assumed to continually update the $V N E$ of its current location.

\section{CONCLUSIONS}

Future work/extensions: In our algorithm each virtual node $V N_{h}, h \in \mathcal{H}$, uses only local information about the target curve $\Gamma$. We can consider a problem extension where the curve is dynamically changing. The curve (or point, even) could be moving targets being tracked. In this case, the coordination of nodes we talked about here is important for two big reasons: (1) maintaining alive $V N \mathrm{~s}$ to detect targets and (2) guiding physical nodes to the moving targets. The fact that we employed a local solution here for curve discovery should adapt well to this more dynamic problem.

It would be possible to modify our algorithm to allow shorter rounds that don't require completed relocation of client nodes; instead we could, for example, have $V N \mathrm{~s}$ update their neighboring region $V N \mathrm{~s}$ of the client nodes that are currently in transit to them.

\section{REFERENCES}

[1] H. Ando, Y. Oasa, I. Suzuki, and M. Yamashita. Distributed memoryless point convergence algorithm for mobile robots with limited visibility. IEEE Transactions on Robotics and Automation, 15(5):818-828, 1999.

[2] Jorge Cortes, Sonia Martinez, Timur Karatas, and Francesco Bullo. Coverage control for mobile sensing networks. IEEE Transactions on Robotics and Automation, 20(2):243-255, 2004. 
[3] Shlomi Dolev, Seth Gilbert, Limor Lahiani, Nancy Lynch, and Tina Nolte. Virtual stationary automata for mobile networks. Technical Report MIT-LCS-TR-979, 2005.

[4] Shlomi Dolev, Seth Gilbert, Nancy Lynch, Alex Shvartsman, and Jennifer Welch. Geoquorums: Implementing atomic memory in mobile ad hoc networks. In 17th International Symposium on Distributed Computing (DISC), 2003.

[5] Shlomi Dolev, Seth Gilbert, Nancy Lynch, Alex Shvartsman, and Jennifer Welch. Geoquorums: Implementing atomic memory in mobile ad hoc networks. Technical Report MIT-LCS-TR-900, 2003.

[6] Shlomi Dolev, Seth Gilbert, Nancy A. Lynch, Elad Schiller, Alexander A. Shvartsman, and Jennifer L. Welch. Virtual mobile nodes for mobile ad hoc networks. In 18th International Symposium on Distributed Computing (DISC), pages 230-244, 2004.

[7] V. Gazi and K. M. Passino. Stability analysis of swarms. IEEE Transactions on Automatic Control, 48(4):692-697, 2003.

[8] David Kiyoshi Goldenberg, Jie Lin, and A. Stephen Morse. Towards mobility as a network control primitive. In MobiHoc '04: Proceedings of the 5th ACM international symposium on Mobile ad hoc networking and computing, pages 163-174. ACM Press, 2004.

[9] A. Jadbabaie, J. Lin, and A. S. Morse. Coordination of groups of mobile autonomous agents using nearest neighbor rules. IEEE Transactions on Automatic Control, 48(6):988-1001, 2003.

[10] J. Lin, A. Morse, and B. Anderson. Multi-agent rendezvous problem. In 42nd IEEE Conference on Decision and Control, 2003.

[11] Nancy Lynch, Roberto Segala, and Frits Vaandrager. Hybrid I/O automata. Information and Computation, 185(1):105-157, August 2003.

[12] Nancy A. Lynch. Distributed Algorithms. Morgan Kaufman, 1996.

[13] Sonia Martinez, Jorge Cortes, and Francesco Bullo. On robust rendezvous for mobile autonomous agents. In IFAC World Congress, Prague, Czech Republic, 2005. To appear.

[14] Michael Merritt, Francemary Modugno, and Mark Tuttle. Time constrained automata. In 2nd International Conference on Concurrency Theory (CONCUR), 1991.

[15] I. Suzuki and M. Yamashita. Distributed autonomous mobile robots: Formation of geometric patterns. SIAM Journal of computing, 28(4):1347-1363, 1999. 


\section{APPENDIX}

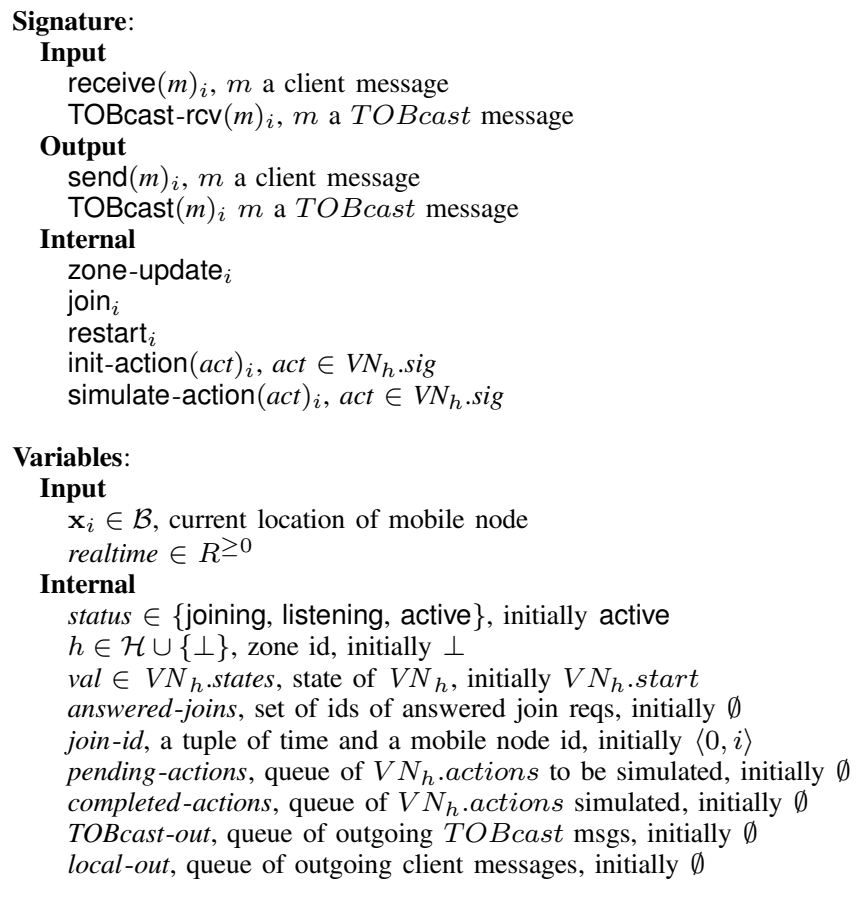

Fig. 9. Signature and variables of $V N E_{i, h}$ algorithm implementing $V N_{h}$.
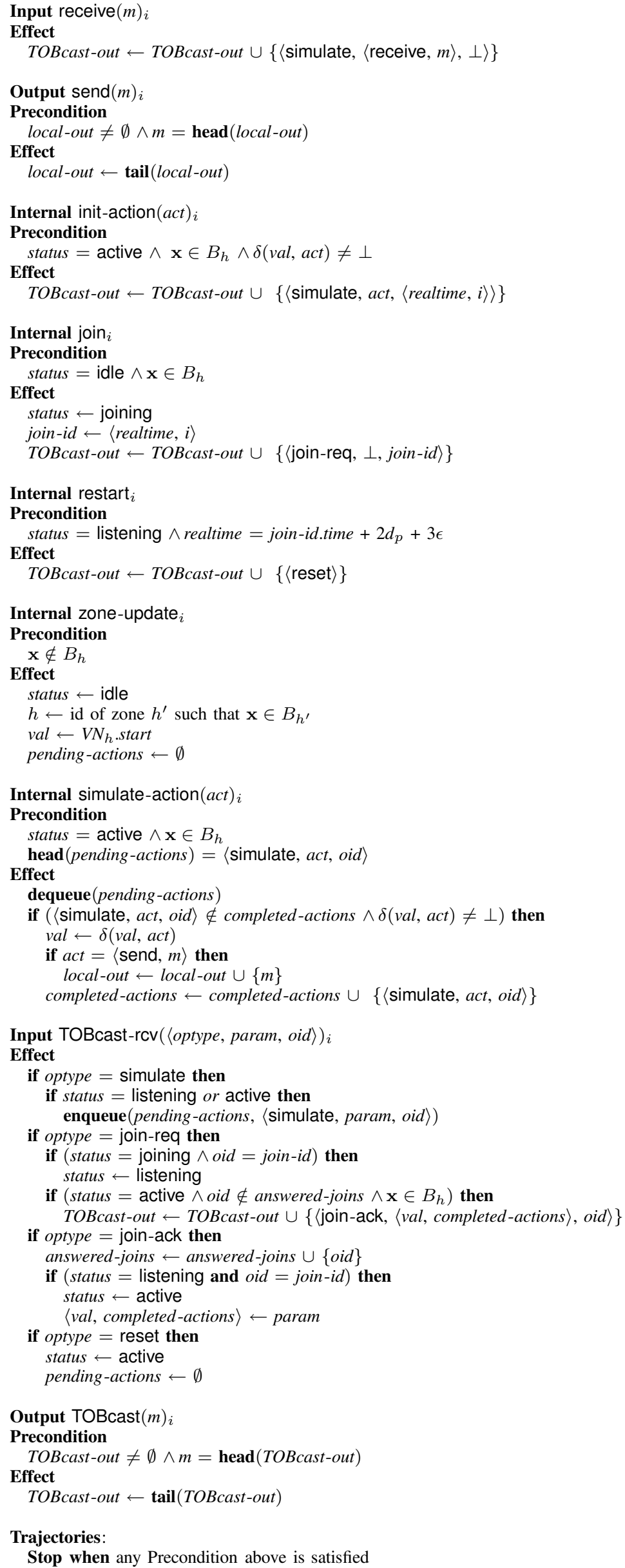

Fig. 10. Transitions and trajectories of $V N E_{i, h}$ algorithm. 